\title{
Isolation of New Self-Cleaving Ribozymes with in vitro Selection
}

\author{
Bongrae Cho* and Younghoon Lee ${ }^{\dagger}$ \\ Department of Applied Chemistry, Division of Applied Science, Cheongju University, Cheongju 360-764, Korea \\ *E-mail:brcho@cju.ac.kr \\ ${ }^{\dagger}$ Dept. of Chemistry and Center for Molecular Design and Synthesis, KAIST, Daejeon 305-701, Korea \\ Received October 7, 2005
}

\begin{abstract}
In vitro selection was used to isolate $\mathrm{Mg}^{2+}$-dependent self-cleaving ribozymes with cis-cleavage activity from a pre-tRNA library having 40-mer random sequences attached to 5'-end of $E$. coli tRNA $^{\text {Phe }}$. After 8 rounds of SELEX (Systematic Evolution of Ligands by Exponential Enrichment), RNA molecules which can self-cleave at the high concentration of $\mathrm{Mg}^{2+}$ were isolated. The selected ribozymes can carry out the self-cleavage reaction in the presence of $100 \mathrm{mM} \mathrm{Mg}^{2+}$ but not in $10 \mathrm{mM} \mathrm{Mg}^{2+}$. The cleavage sites of the ribozymes are located at +3 and +4 of tRNA $^{\text {Phe }}$, compared with +1 position of 5'-end cleavage site of pre-tRNA by RNase P. New RNA constructs deprived of its D stem-loop, anticodon stem-loop, variable loop and $\mathrm{T}$ stem-loop, respectively showed the cleavage specificity identical to a ribozyme having the intact tRNA structure. Also, the new ribozyme fused with both a ribozyme and $\mathrm{RRNA}^{\mathrm{Leu}}$ showed the cleavage activities at the various sites within its sequences, different from two sites of position +3 and +4 observed in the ribozyme with tRNA ${ }^{\text {Phe }}$. Our results suggest that the selected ribozyme is not structural-specific for tRNA.
\end{abstract}

Key Words : SELEX, Self-cleavage ribozyme, tRNA, H1 RNA

\section{Introduction}

Natural ribozymes such as the hammerhead, hairpin, hepatitis delta virus and Neurospora VS, are RNA molecules capable of self-cleavage reaction at a unique site within their sequences to yield a 5' hydroxyl and a 2',3' cyclic phosphodiester as product. ${ }^{1}$ These ribozymes discovered in biological systems are essential to cellular life such as included in the control of gene expression. ${ }^{2}$ Nowadays there have been many attempts to modify and improve the activities of natural self-cleaving ribozymes because of the functional limitations of them and to isolate new small functional RNAs by in vitro evolution., ${ }^{3,4}$ These attempts make us find new artificial ribozyme with more improved specificity rather than natural ribozymes or absolutely new ribozymes which are not found in nature.

RNase $\mathrm{P}$ is involved in generating the mature $5^{\prime}$-end of tRNAs by a single endonucleolytic cleavage of their precursors. It is an essential, ubiquitous enzyme in all cells and cellular compartments that synthesize tRNA: archaea, bacteria, eucarya, mitochondria and chloroplasts. All known RNase $\mathrm{P}$ enzymes are ribonucleoproteins and an RNA subunit remains the catalytic center of them. ${ }^{6,7}$ E. coli RNase $\mathrm{P}$ consists of catalytically active M1 RNA (377 nucleotides) and cofactor, C5 protein (119 amino acids). M1 RNA alone can be catalytically active in the presence of high concentration of a divalent cation, especially $\mathrm{Mg}^{2+} .8$ By contrast, RNase P RNAs from eukaryotes and archaebacteria have not been demonstrated to exhibit enzymatic activity so far in the absence of their own protein components and have significantly high protein content in the holoenzyme. ${ }^{9,10,11,12}$ Why is eukaryotic RNase P RNA catalytically inactive even in the presence of high concentration of a divalent cation unlike
M1 RNA? To answer this question, it is necessary to know whether eukaryotic RNase P RNA needs extra sequence motifs for its in vitro enzymatic activity in the presence of high concentration of $\mathrm{Mg}^{2+}$ ion without its protein components.

In this study, we set out an in vitro evolution with a pretRNA library having random sequences attached to 5 '-end of E. coli tRNA ${ }^{\text {Phe }}$ to look for the extra sequence motifs for the in vitro enzymatic activity of human RNase P RNA (H1 RNA) in the presence of high concentration of $\mathrm{Mg}^{2+}$ ion.

\section{Experimental Section}

Preparation of a DNA library. Double-strand DNA library was prepared through one cycle of RCR with a DNA primer of 72-mer (AGGGAGGACGATGCGG-N40-CAGACGACGAGCGGGA; $N$ represents an equal mixture of the four nucleotides) and revtRNA primer of 92-mer (TGGTGCCCGGACTCGGAATCGAACCAAGGACACGGGGAT TTTCAATCCCCTGCTCTACCGACTGAGCTATCCGGG GCTCCCGCTCGTCGTCTG), and amplified through six cycles of PCR using 5' random primer (GAAATTAATACGACTCACTATAGGGAGGACGATGCGG; T7 promoter is underlined) and 3 ' tRNA primer (TGGTGCCCGGACTCGG) (Fig. 1).

In vitro selection. The RNA population was created from the corresponding DNA library with T7 RiboMax Large Scale RNA Production System (Promega, Madison, WI) in a 20-uL reaction according to the manufacturer's directions, purified by a denaturing $8 \%$ PAGE, and isolated from the gel by crush-soaking in elution buffer (10 mM Tris-Cl, $\mathrm{pH} 8.0$ / $0.3 \mathrm{M} \mathrm{NaCl} / 1 \mathrm{mM}$ EDTA). Purified RNAs were precipitated from the solution by the addition of sodium acetate to 0.3 

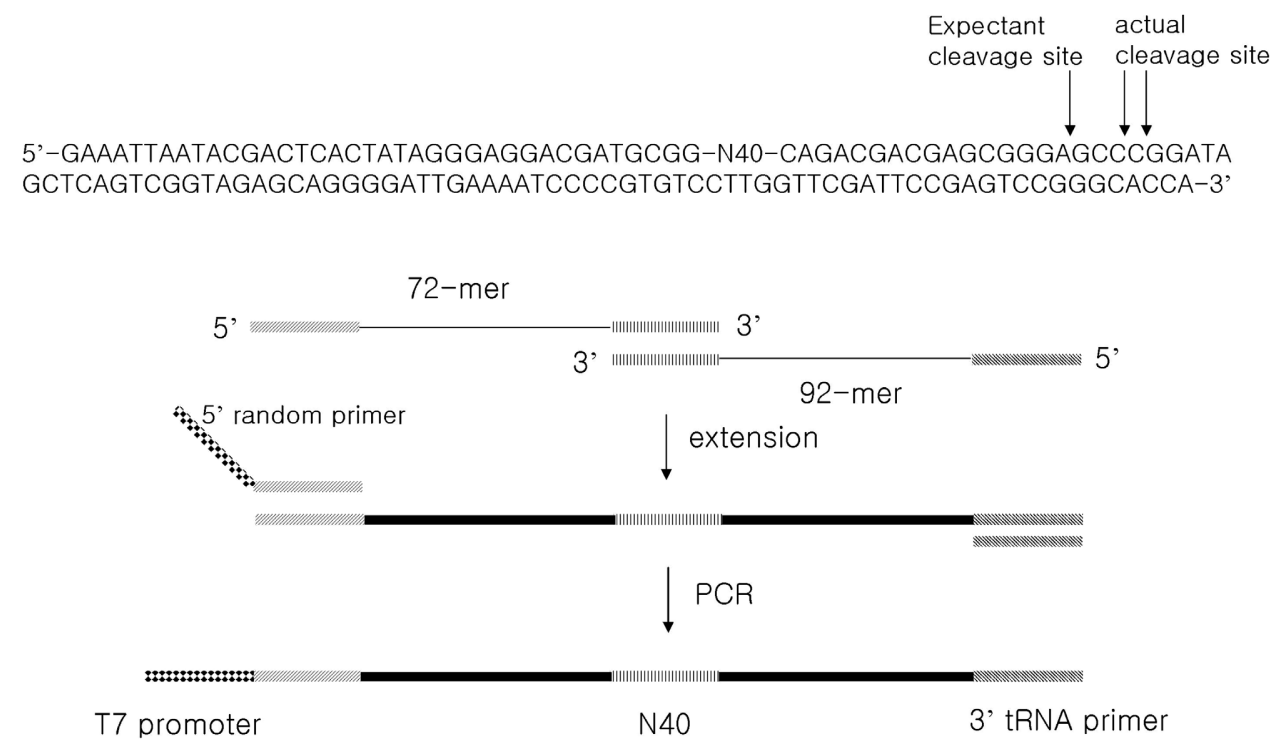

Figure 1. DNA construct used in this experiment for in vitro selection. The sites of cleavage expected and observed in this experiment are indicated by arrows. N40 shows 40 random-sequence nucleotides. Double-strand DNA library was prepared through one cycle of PCR with a DNA primer of 72-mer having 40 random sequence and revtRNA primer of 92-mer having tRNA ${ }^{\text {Phe }}$ sequence during the extension, and amplified using both 5' random primer having T7 promoter and 3' tRNA primer.

$\mathrm{mM}(\mathrm{pH} 5.2)$ and 2.5 volume of $100 \%$ ethanol. The precipitated library RNAs were preincubated in high salt buffer (50 $\mathrm{mM}$ Tris-Cl, $\mathrm{pH} 8.0 / 100 \mathrm{mM} \mathrm{NH}_{4} \mathrm{Cl} / 100 \mathrm{mM} \mathrm{MgCl}$ ) at 37 ${ }^{\circ} \mathrm{C}$ for $10 \mathrm{~min}$ and cooled to room temperature. The cleavage reaction was initiated by the addition of human RNase $\mathrm{P}$ RNA (H1 RNA). The same reaction was carried out with library RNAs by M1 RNA for the comparison of the location of the RNA cleavage products. The samples were loaded on a denaturing $8 \%$ polyacrylamide gel and the desired RNA cleavage products were eluted from the gel as above. Eluted RNAs were reverse-transcribed with revtRNA primer to generate complementary DNAs, which were then amplified by PCR with $5^{\prime}$ random primer and 3' tRNA primer. The resulting double-stranded DNA was used to transcribe the subsequent RNA population. After eight rounds of selection, the cleavage products isolated by PAGE were amplified by RT-PCR. Amplified DNA fragments were ligated into pGEM-T Easy vector and transformed into E. coli BL21 competent cells. Sequences of the insertions identified with restriction endonuclease $E c o$ R I were determined.

Cleavage sites for unimolecular reaction. 5'-end radiolabeling of RNAs dephosphorylated with CIAP was carried out using T4 polynucleotide kinase and $\left[\chi^{32} \mathrm{P}\right] \mathrm{ATP}$, and the reaction products were purified by a denaturing $5 \%$ PAGE. 5'-end labeled ribozyme was incubated at the 100 $\mathrm{mM}$ of or $10 \mathrm{mM}$ of $\mathrm{Mg}^{2+}$ ion under the condition of high salt buffer. The cleavage fragments were separated by a denaturing $8 \%$ PAGE and their locations were compared with those of cleavage fragments generated by partial RNA digestion using RNase $\mathrm{T} 1$ and alkali.

Ribozyme truncations. The D stem-loop deletions were made from the extension reaction with ribozyme 1 primer of 93-mer (GAAATTAATACGACTCACTATAGGGAGGACGATGCGGTCTTCACACCGTACTGCAGTTTTGGGCTC-
TTTGTGCTAGTCAGACGACGAGCGGGA) and D primer of 76-mer (TGGTGCCCGGACTCGGAATCGAACCAAGGACACGGGGATTTTCAATCCCCTTATCCGGGCTCCCGCTCGTCGTCTG). The anticodon stem-loop deletions were made with ribozymel primer and anticodon primer of 75-mer (TGGTGCCCGGACTCGGAATCGAACCAAGGACACTGCTCTACCGACTGAGCTATCCGGGCTCCCGCTCGTCGTCTG). The variable loop deletions were with ribozyme1 primer and variable primer of 87-mer (TGGTGCCCGGACTCGGAATCGAACCAAGGGGGATTTTCAATCCCCTGCTCTACCGACTGAGCTATCCGGGCTCCCGCTCGTCGTCTG). The T stem-loop deletions were made with ribozymel primer and $\mathrm{T}$ primer of 75 -mer (TGGTGCCCGGAGACACGGGGATTTTCAATCCCCTGCTCTACCGACTGAGCTATCCGGGCTCCCGCTCGTCGTCTG). Each DNA template was amplified by PCR with $5^{\prime}$ random primer and $3^{\prime}$ tRNA primer. Specific truncated RNAs were generated by in vitro transcription using T7 RNA polymerase and the corresponding template DNAs, and purified by a denaturing $8 \%$ PAGE, dephosphorylated and $5^{\prime}{ }^{32} \mathrm{P}$-labeled. The self-cleavage reactions of the truncated ribozyme were conducted as described previously.

Fusions of a ribozyme and $t R N A^{\text {Leu. The fusions were }}$ made with an ribozyme1 primer and the primer of 103-mer corresponding to tRNA ${ }^{\text {Leu }}$ (TGGTGCGAGGGGGGGGACTTGAACCCCCACGTCCGTAAGGACACTAACATCTGAAGCTAGCGCGTCTACCAATTCCGCCACCTTCGCTCCCGCTCGTCGTCTG). DNA template was amplified by PCR with $5^{\prime}$ random primer and $3^{\prime}$ tRNA ${ }^{\mathrm{Leu}}$ primer of 16 mer (TGGTGCGAGGGGGGGG). Fused RNAs were generated by $\mathrm{T} 7$ in vitro transcription system and purified by a denaturing $8 \%$ PAGE, dephosphorylated and $5^{\prime}{ }^{32} \mathrm{P}$-labeled. The self-cleavage reactions of the fused ribozyme were conducted as described previously. 


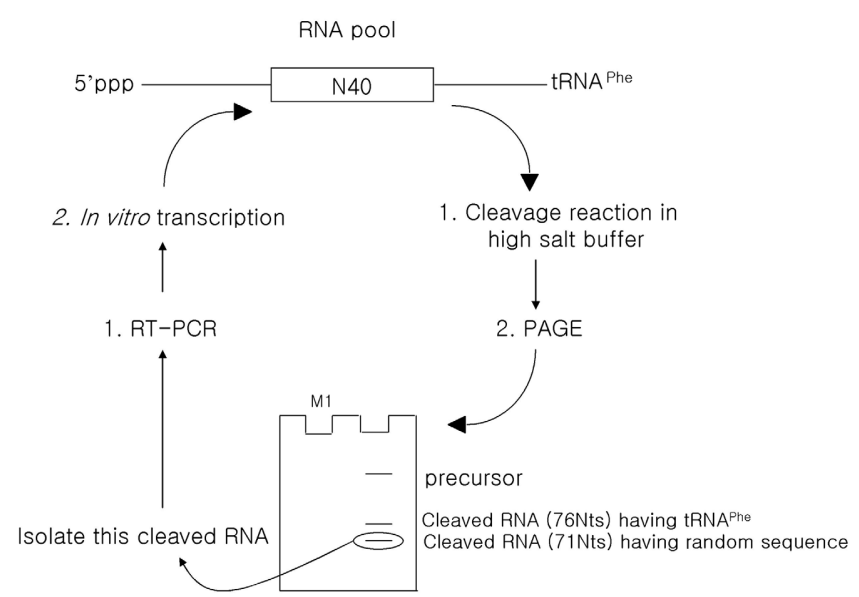

Figure 2. SELEX Scheme of self-cleaving ribozymes. The RNA library having random sequences attached to 5 '-end of $E$. coli tRNA $^{\text {Phe }}$, was incubated in high salt buffer condition with $100 \mathrm{mM}$ $\mathrm{Mg}^{2+}$. The RNA cleavage products were separated from uncleaved precursors by a denaturing $8 \%$ PAGE and their positions were identified with the comparison of reaction products of M1 RNA (denoted with M1). The desired RNA cleavage products with 71 nucleotides were eluted from the gel by crushing-soaking and, reverse-transcribed to generate cDNA, which were then amplified by PCR. The resulting double-strand DNAs were used to transcribe the subsequent RNA population in vitro for the next round of selection.

\section{Results and Discussion}

An in vitro selection was applied to look for the extra RNA motifs for the in vitro enzymatic activity of human RNase P RNA (H1 RNA) in the presence of $100 \mathrm{mM} \mathrm{Mg}^{2+}$ from a population of $\sim 10^{14}$ different RNAs having 40-mer random sequences attached to 5'-end of E. coli tRNA $^{\text {Phe }}$ which serves as substrate but only new self-cleavage ribozymes were selected. The fragments self-cleaved from RNA library transcribed in vitro using T7 RNA polymerase were thought to be 76-mers corresponding to tRNA ${ }^{\text {Phe }}$ and 71-mer of RNA molecules which were amplified by RT-PCR (Fig. 2).

The bands of the population of RNAs isolated in the beginning of SELEX could not be seen on the polyacryl-

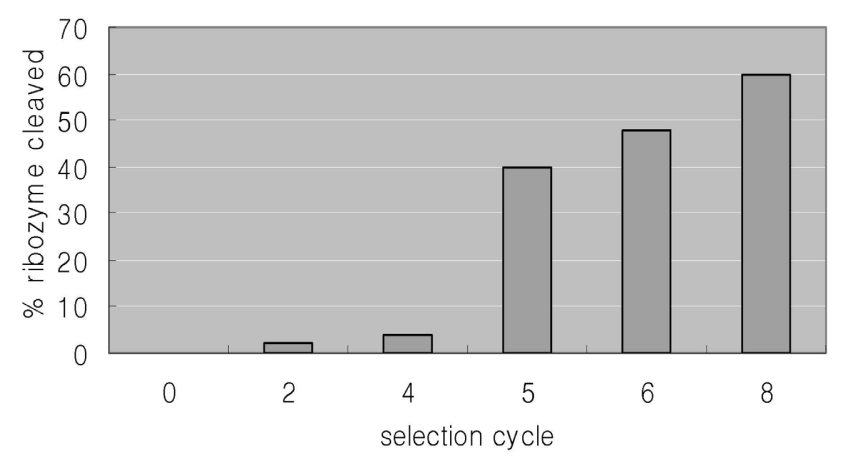

Figure 3. Comparison of the ratio of ribozyme pools cleaved at different numbers of rounds of SELEX. The fraction of the cleaved ribozymes is low for the first four cycles, then jumps in cycle five.

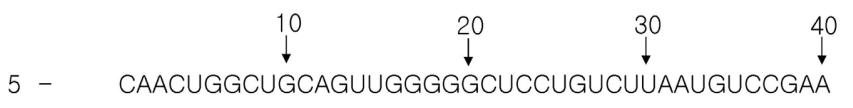

10- UUUCACACCGUGCUGCAGUUUAGGGCUCUUUGUGCUAGU

11- UCUUCACACCGUAUUGCAGUUUUGGACUCUUUGUGCUAGU

1 - UCUUCACACCGUACUGCAGUUUUGGGCUCUUUGUGCUAGU

2 - UCUUCACACCGUACUGCAGUUUUGGGCUCUUUGUGCUAGU

4 - UCUUCACACCGUACUGCAGUUUUGGGCUCUCUGUGCUAGU

7 - UCUUCACACCGUACUGCAGUUUUGGGCUCUUUGUGCUAGU

12- UAUCUGUUCUACCGAUUGCAGGCAGGGCCCCGGAUUUGU

3 - UAUCUGUUCUACCGAUUGCGGGCAGGGCCCCGGAUUUGU

6 - UAUCUGUUCUACCGAUUGCAGGCAGGGCCCCGGAUUUGU

8 - UAUCUGUUCUACCGAUUGCAGGCAGGGCCCCGGAUUUGU

9 - UAUCUGUUCUACCGAUUGCAGGCAGGGCCCCGGAUUUAU

Figure 4. Sequences of RNA molecules selected. After 8 rounds of SELEX, The selected RNA molecules were amplified to DNAs by RT-PCR, which were ligated to pGEM-T Easy vector. The chimeric plasmid was then used to transform $\mathrm{Ca}^{2+}$-treated heatshocked E. coli cells. Sequences of the insertions were determined by the chain termination method. The different bases in each group are underlined.

amide gel but could be seen after five rounds of selection (Fig. 3). The populations derived from total 8 rounds of SELEX after three more rounds of reselection were selectively recovered from the gel and identified as having selfcleavage activity irrespective of the presence of H1 RNA. In other words, new RNA molecules with self-cleavage activity and independent of H1 RNA were selected. Therefore, we conclude that we could not get the RNA motifs to help H1 RNA to have RNase $\mathrm{P}$ activity in vitro. The protein components are thought to be necessary for its ribozyme activity in the light of the fact that RNase P RNAs from eukaryotes have very high protein content in the holoenzyme., ${ }^{9,10}$ The selected populations were subject to RT-PCR amplification followed by cloning and sequencing (Fig. 4).

Sequence analysis of clones revealed 12 ribozyme sequences which were grouped into 3 classes based on the sequence similarity. The cleavage sites were determined with ribozyme 1 and 8 which had many identical or similar sequences among the populations. Ribozyme 1 cleaved at two different sites of position 74 and 75 , which are inside the position 71 , the $5^{\prime}$ end of $\mathrm{RNA}^{\text {Phe }}$ (Fig. 5). Their cleavage sites are located at +3 and +4 of $\mathrm{tRNA}^{\text {Phe }}$, compared with +1 position of 5'-end cleavage site of pre-tRNA by RNase P. This ribozyme is thought to do the self-cleavage reaction with a mechanism different from human RNase P RNA because it has the cleavage activity even in the absence of H1 RNA. Therefore, it is thought that the cleavage sites of this selected ribozyme are different from that of pre-tRNA by RNase P. It is interesting for these cleavage sites to locate in the double-strand rather than single-strand region of the secondary structures predicted with the algorism RNA mfold accessed on the internet (www.bioinfo.rpi.edu/ applications $/ \mathrm{mfold} / \mathrm{old} / \mathrm{rna}$ ). But the structural analysis using enzymes and chemicals needs to be applied for the accurate structure of the cleavage sites. The same cleavage sites were observed with ribozyme 8 , a representative of one of three groups (data not shown). The band denoted with * at the low 


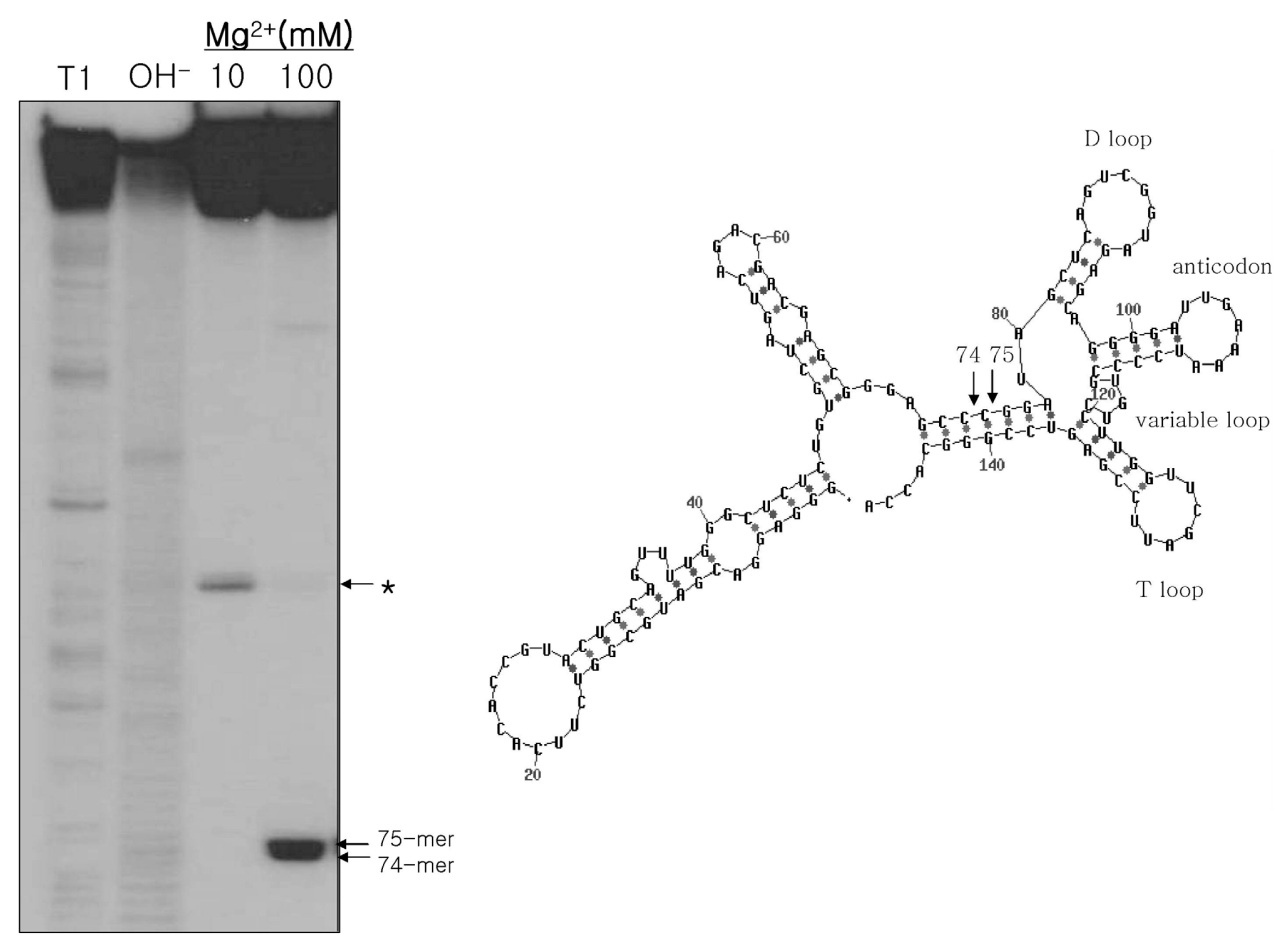

Figure 5. Determination of cleavage sites for a cis-acting ribozyme. $5^{\prime}{ }^{32} \mathrm{P}-$ labeled ribozyme was incubated with $10 \mathrm{mM} \mathrm{Mg}^{2+}$ and $100 \mathrm{mM}$ $\mathrm{Mg}^{2+}$. The cleavage fragments were separated by a denaturing $8 \%$ PAGE and their sizes were compared with fragments prepared by partial ribonuclease (T1) and partial alkaline $\left(\mathrm{OH}^{-}\right)$digests. The two cleaved fragments having 74 and 75 nucleotides are indicated by arrows. The secondary structure was predicted with the algorism RNA mfold accessed on the internet (www. bioinfo.rpi.edu/applications/mfold/old/rna).

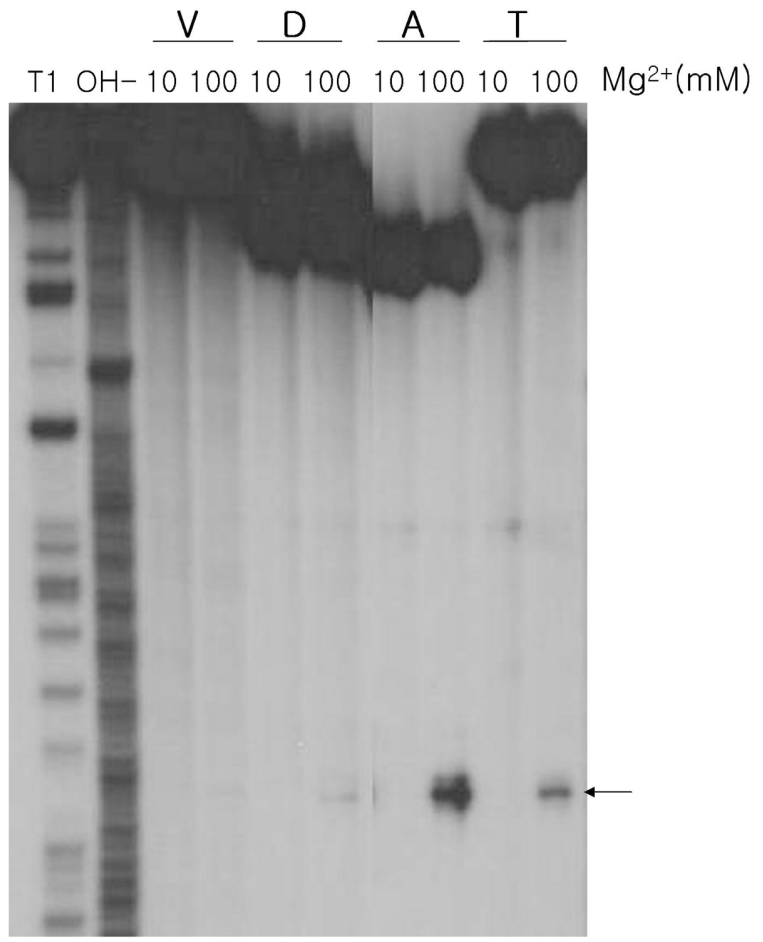

Figure 6. Determinations of cleavage sites for truncated ribozymes. $\mathrm{V}, \mathrm{D}, \mathrm{A}$ and T identify new RNA constructs deprived of its variable loop, D stem-loop, anticodon stem-loop and T stem-loop, respectively. Other notations are as described in the legend to Figure 5.

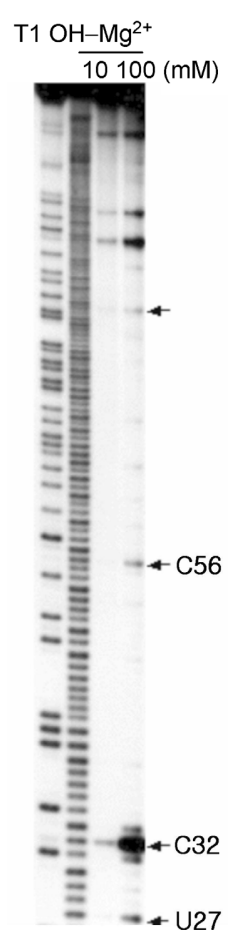

Figure 7. Determinations of cleavage sites for a ribozyme fused with a selected ribozyme and tRNA ${ }^{\text {Leu }}$. The cleavage sites are indicated by arrows. Notations are as described in the legend to Figure 5. 
concentration of $10 \mathrm{mM} \mathrm{Mg}{ }^{2+}$ in Figure 5 is thought be involved in the binding of $\mathrm{Mg}^{2+}$. In fact, $\mathrm{Mg}^{2+}$ participates in binding to phosphate oxygens and bases in yeast tRNA $^{\text {Phe }} \cdot{ }^{13,14}$

To know which part of $\mathrm{tRNA}^{\text {Phe }}$ is important for the cleavage reaction, we made new RNA constructs deprived of its D stem-loop, anticodon stem-loop, variable loop and T stem-loop, respectively. The variable loop deletions and the D stem-loop deletions showed weak cleavage reactions, compared with a selected ribozyme having the intact tRNA structure but the anticodon stem-loop deletions and the $\mathrm{T}$ stem-loop deletions showed strong cleavage reaction (Fig. 6). Our results indicate that new RNA constructs have the cleavage specificity identical to a ribozyme having the intact tRNA structure but their cleavage activities are more dependent on the structure of the anticodon stem-loop and the T stem-loop rather than the variable loop and the D stemloop.

We fused a ribozyme 1 and $\mathrm{RNNA}^{\mathrm{Leu}}$ which has a variable loop of 15 nucleotides to know that the nucleotide number of the variable loop is important to cleavage reaction. The variable loop of tRNA $^{\text {Phe }}$ used in this selection, has 5 nucleotides. The fusions showed the cleavage activities at the various sites within its sequences, different from two sites of position 74 and 75 observed in the ribozyme with tRNA $^{\text {Phe }}$ (Fig. 7). Our results suggest that the selected ribozyme is not structure-specific for tRNA.

Acknowledgment. We thank Dr. Jaehyeong Ko at the department of Chemistry, KAIST for his helpful observation and discussion.

\section{References}

1. Gesteland, R. F.; Cech, T. R.; Atkins, J. F. In The RNA World, 2nd ed; Cold Spring Harbor Laboratory Press: Cold Spring Harbor, New York, 1999; pp 265-286.

2. Winkler, W. C.; Nahvi, A.; Roth, A.; Collins, J. A.; Breaker, R. R. Nature 2004, 428, 281-286.

3. Tang, J.; Breaker, R. R. Proc. Natl. Acad. Sci. USA 2000, 97, 5784-5789.

4. Meli, M.; Vergne, J.; Maurel, M.-C. J. Biol. Chem. 2003, 278 , 9835-9842.

5. Zamel, R.; Poon, A.; Jaikaran, D.; Andersen, A.; Olive, J.; Abreu, D. D.; Collins, R. A. Proc. Natl. Acad. Sci. USA 2004, 101, $1467-$ 1472.

6. Frank, D. N.; Pace, N. R. Annual Review Biochem. 1998, 67, $153-$ 180.

7. Gopalan, V.; Vioque, A.; Altman, S. J. Biol. Chem. 2002, 277, 6759-6762.

8. Guerrier-Takada, C.; Haydock, K.; Allen, L.; Altman, S. Biochemistry 1986, 25, 1509-1515.

9. Akaboshi, E.; Guerrier-Takada, C.; Altman, S. Biochem. Biophys. Res. Comm. 1980, 96, 831-837.

10. Morales, M. J.; Dang, Y. L.; Lou, Y. C.; Sulo, P.; Martin, N. C. Proc. Natl. Acad. Sci. USA 1992, 89, 9875-9879.

11. Altman, S.; Kirsebom, L.; Talbot, S. FASEB J. 1993, 7, 7-14.

12. Jarrous, N.; Eder, P. A.; Guerrier-Takada, C.; Hoog, C.; Altman, S. RNA 1998, 4, 407-417.

13. Holbrook, S. R.; Sussman, J. L.; Warrant, W. R.; Church, G. M.; Kim, S.-H. Nucleic Acids Res. 1977, 4, 2811.

14. Quigley, G. J.; Teeter, M. M.; Rich, A. Proc. Natl. Acad. Sci. USA 1978, 75, 64 . 